\title{
Tornado funnel-shaped cloud as convection in a cloudy layer
}

\author{
M. V. Zavolgenskiy ${ }^{1}$ and P. B. Rutkevich ${ }^{2}$ \\ ${ }^{1}$ Water Problems Institute RAN, South Department, RAN, 198 Stachka av., Rostov-on-Don, Russia \\ ${ }^{2}$ Space Research Institute (IKI) RAN, Moscow, Russia
}

Received: 29 December 2008 - Revised: 2 February 2009 - Accepted: 15 March 2009 - Published: 2 April 2009

\begin{abstract}
Analytical model of convection in a thick horizontal cloud layer with free upper and lower boundaries is constructed. The cloud layer is supposed to be subjected to the Coriolis force due to the cloud rotation, which is a typical condition for tornado formation. It is obtained that convection in such system can look as just one rotating cell in contrast to the usual many-cells Benard convection. The tornado-type vortex is different from spatially periodic convective cells because their amplitudes vanish with distance from the vortex axis. The lower boundary at this convection can substantially move out of the initially horizontal cloud layer forming a single vertical vortex with intense upward and downward flows. The results are also applicable to convection in water layer with negative temperature gradient.
\end{abstract}

\section{Introduction}

A concept first proposed by Bergeron (1959) states that our understanding or nature moves forward most rapidly when observations, tools, and models are being advanced simultaneously and interactively. By models Bergeron meant not just numerical models, but also dynamical, statistical and conceptual ones. Indeed, every atmosphere event is unique, but it has many common aspects with other events of the same nature. Without both a detailed understanding of storms available in a research mode, and a large sample size, it is going to be difficult to synthesize comprehensively new understanding.

Detailed observations of the Oklahoma tornado storms of 26 May 1963 formed the basis for an important summary publications by Browning $(1962,1964)$, which had considerable importance in establishing a concept now known as supercell convection. This conceptual model was deduced from radar measurements, inferring internal storm airflows from the structure and evolution of the radar-detectable precipitation echoes.

Modelling tornado as a convective cell is not unique. For example, Emanuel and Bister (1996), and Renno (1996, 1998), considered atmospheric convection as reversible heat engines. In paper Souza et al. (2000) the heat engine frame

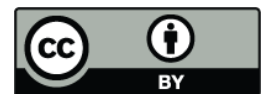

Correspondence to: P. Rutkevich (pbrutkevich@gmail.com) work is used to formulate a theory for circulations driven by surface heterogeneities in sloping terrains. Dowell et al. (2005) considers how the objects in a tornado respond to a specific vortex flow. Wicker and Wilhelmson (1995) consider a three-dimensional numerical simulation of tornado genesis within a supercell.

The convective supercell concept proved to be useful. But on the other hand it is well known, that the theory of convection does not describe localized structures.

In the present paper we consider rotating cloud layer in unstable (convective) conditions with both (upper and lower) movable boundaries. It is expectable, that vertical air flow has to affect the structure of the convective layer itself. We are going to find out whether a local solution can exist in such system, and to investigate the stationary shape of the boundaries.

We assume, that due to heat exchange between underlying surface and upper atmosphere the cloud layer appears with negative (convective) vertical temperature gradient. A similar situation can appear in the ocean due to stream mixing. A well known condition preventing formation of convective stability corresponds to a linear temperature profile with low value of vertical temperature gradient Chandrasekhar (1961); Landau and Lifshitz (1987), while temperature gradient exceeding the threshold value corresponds to convective instability. For large-scale processes, like hurricane or tornado, rotation of the system introduces strong Coriolis force in the system, which must be taken into account. 


\section{Formulation of the problem}

We introduce cylindrical system of coordinates $\left(r^{\prime}, \varphi, z^{\prime}\right)$ with $z^{\prime}=0$ at the bottom edge of the layer. We assume that the fluid layer occupies the region $0 \leq z^{\prime} \leq h$ and $0 \leq r^{\prime}<\infty$, and has temperature difference $\vartheta^{\prime}>0$ between its top and bottom surfaces. The entire system is rotating at constant angular velocity $\omega^{\prime}$, under constant gravity $\mathbf{g}$. The fluid is incompressible, all parameters, namely, viscosity $v$, density $\rho$, coefficient of thermal expansion $\beta$, thermal conductivity $\lambda$, and heat capacity $c$ are constant. We also assume that pressure $p_{1}\left(r^{\prime}, z^{\prime}\right)$ along the lower edge of the layer is constant. Throughout the paper indices 1 and 2 stand for the bottom and top surfaces of the layer, respectively. Positions of the top $\left(\zeta_{2}^{\prime}\right)$ and bottom $\left(\zeta_{1}^{\prime}\right)$ edges of the layer are to be determined. This method was successfully used by Rutkevich et al. (1989), who studied interaction between surface and internal waves in stratified ocean. In that paper the layer had only one boundary (the ocean surface) and two horizontal coordinates, while in the present paper the layer has two boundaries and is axisymmetric.

The complete set of equations becomes now:

$$
\begin{aligned}
& \frac{\partial \mathbf{v}^{\prime}}{\partial t^{\prime}}+\left(\mathbf{v}^{\prime} \cdot \nabla\right) \mathbf{v}^{\prime}+2 \omega^{\prime} \times \mathbf{v}^{\prime}=-\frac{\nabla p^{\prime \prime}}{\rho}-\beta T^{\prime} \mathbf{g}+\nu \nabla^{2} \mathbf{v}^{\prime}, \\
& \nabla \cdot \mathbf{v}^{\prime}=0, \quad \rho c\left(\frac{\partial T^{\prime}}{\partial t^{\prime}}+\mathbf{v}^{\prime} \cdot \nabla T^{\prime}\right)=\lambda \nabla^{2} T^{\prime}+\mu \Phi,
\end{aligned}
$$

where pressure $p^{\prime \prime}=p^{\prime}-p_{1}+\rho g z^{\prime}$, velocity is presented in cylindrical components $\mathbf{v}^{\prime}=\left\{v_{r}, v_{\varphi}, v_{z}\right\}$ and the Hamilton operator $\nabla$ also has to be written in cylindrical coordinates.

Assuming negligible friction between the convective layer and the surrounding fluid, the stress tensor can be diagonalized: the orthogonal components are constant and equal to the external pressure at the corresponding side of the layer $p_{n n}=-p_{j}$, and the stress parallel to the surface is equal to zero: $p_{n \tau}=p_{n s}=0$. Here $\mathbf{n}, \boldsymbol{s}$ and $\boldsymbol{\tau}$ are the vector perpendicular to the surface, and the two orthogonal directions along the surface, respectively.

The boundary conditions can be written as follows:

$$
\begin{aligned}
& T^{\prime}=\vartheta_{j}^{\prime}, \frac{\partial \zeta_{j}^{\prime}}{\partial t^{\prime}}+\mathbf{v}^{\prime} \cdot \nabla \zeta_{j}^{\prime}=v_{z} \quad\left(z^{\prime}=\zeta_{j}^{\prime}\right), \\
& p_{2}=p_{1}+\rho g h\left(1+\frac{1}{2} \beta \vartheta^{\prime}\right), \quad \vartheta_{1}^{\prime}=0, \quad \vartheta_{2}^{\prime}=\vartheta^{\prime}, \\
& \mathbf{v}^{\prime}=0, \quad T^{\prime}=T_{0}^{\prime} \quad\left(r^{\prime}=\infty\right), \quad j=1 ; 2,
\end{aligned}
$$

The solution of system (1)-(5) has to vanish in the limit $r^{\prime} \rightarrow \infty$.

We introduce dimensionless variables, based on physical parameters $v, \beta, h$, and $g$. Let $\varepsilon=v^{2} /\left(g h^{3}\right)$, then

$$
\left\{v_{r}, v_{\varphi}, v_{z}\right\}=\frac{v \varepsilon}{h}\{u, v, w\}, \quad p^{\prime}=p_{1}^{\prime}+\frac{\rho v^{2} \varepsilon}{h^{2}} p,
$$

$$
\begin{aligned}
& \gamma=\frac{\rho v c}{\lambda}, \quad r=\frac{r^{\prime}}{h} s, \\
& T^{\prime}=T_{1}^{\prime}+\frac{\varepsilon^{2}}{\beta} T, \quad \zeta_{1}^{\prime}=\varepsilon^{2} h \zeta_{1}, \quad \zeta_{2}^{\prime}=h\left(1+\varepsilon^{2} \zeta_{2}\right), \\
& z=\frac{z^{\prime}}{h}, \quad t=\frac{v t^{\prime}}{h^{2}} \sigma, \\
& p_{1}^{\prime}=p_{1}-\rho g z^{\prime}-\frac{\rho g \beta \vartheta^{\prime}}{2 h} z^{\prime 2}, \quad T_{1}^{\prime}=-\frac{\vartheta^{\prime}}{h} z^{\prime}, \\
& \vartheta=\frac{\rho g \beta \vartheta^{\prime} c}{\lambda v} h^{3}, \quad \omega=\frac{2 \omega^{\prime} h^{2}}{v} .
\end{aligned}
$$

The final system of equations in dimensionless variables becomes:

$\nabla \cdot \mathbf{v}=0, \quad \sigma \gamma \frac{\partial T}{\partial t}=\nabla^{2} T+\vartheta w$,

$\sigma \frac{\partial \mathbf{v}}{\partial t}=\nabla^{2} \mathbf{v}-\nabla p-\omega \times \mathbf{v}+\mathbf{k} T$,

and boundary conditions:

$w=T=\frac{\partial u}{\partial z}=\frac{\partial v}{\partial z}=0$,

$\zeta_{j}=p-2 \frac{\partial w}{\partial z} \quad(z=0,1 ; j=1,2)$,

$\mathbf{v}(r=\infty)=0, \quad T(r=\infty)=0$,

where $\mathbf{k}=\{0,0,1\}$ is a unit vector along the z-axis. The operator $\nabla$ contains parameter $s$ in front of radial and angular derivatives.

\section{Solution and analysis}

The system (8)-(10) has been solved using separation of variables. We introduce

$$
\begin{array}{r}
\left(u, v, w, p, T(r, \varphi, z, t), \zeta_{j}(r, \varphi, t)\right)= \\
\left(\tilde{U}, \tilde{V}, \tilde{W}, \tilde{P}, \tilde{\Theta}(r, z), Z_{j}(r)\right) e^{i m \varphi+t}
\end{array}
$$

where $j=1,2$; and $m=0, \pm 1, \pm 2, \ldots$ and $i=\sqrt{-1}$ is the imaginary unit.

So the solution takes the form:

$W=A \sin (k \pi z) \quad(k= \pm 1, \pm 2, \ldots)$

where the relation between the amplitude $A$ and the parameter $\sigma$ as a power series with respect to small $\epsilon$ can be found from the condition of solvability of linear inhomogeneous problem.

Values $k$ are related to parameters $\omega, s, \vartheta$ and $\sigma$ through

$$
S \gamma \sigma^{3}+S^{2}(2 \gamma+1) \sigma^{2}+\left[S^{3}(\gamma+1)+\xi_{0}^{2}(\gamma-1)-\frac{\xi}{S}\right] \sigma=\xi,
$$


$\xi=S\left(s^{2} \vartheta-S^{3}-\xi_{0}^{2}\right), S=k^{2} \pi^{2}+s^{2}, \xi_{0}=k \omega \pi$,

$u=\frac{A k \pi}{s}\left[J_{m}^{\prime}(r)+\frac{i m \omega}{r(S+\sigma)} J_{m}(r)\right] e^{\sigma \tau+i m \varphi} \cos k \pi z$,

$v=\frac{A k \pi}{s}\left[\frac{i m}{r} J_{m}(r)-\frac{\omega}{S+\sigma} J_{m}^{\prime}(r)\right] e^{\sigma \tau+i m \varphi} \cos k \pi z$

$w=A J_{m}(r) e^{\sigma \tau+i m \varphi} \sin k \pi z$,

$T=\frac{A \vartheta \sin k \pi z}{S+\sigma \gamma} J_{m}(r) e^{\sigma \tau+i m \varphi}, \quad \tau=\frac{v t^{\prime}}{h^{2}}$

$p=-\frac{A k \pi \cos k \pi z}{s^{2}(S+\sigma)}\left[(S+\sigma)^{2}+\omega^{2}\right] J_{m}(r) e^{\sigma \tau+i m \varphi}$,

$\zeta_{1}=-A k \pi\left[2+\frac{(S+\sigma)^{2}+\omega^{2}}{s^{2}(S+\sigma)}\right] e^{\sigma \tau+i m \varphi} J_{m}(r)$,

$\zeta_{2}=(-1)^{k} \zeta_{1} m=0, \pm 1, \ldots, k= \pm 1, \pm 2, \ldots$

The presented solution is applicable for a wide range of rotating flows in both a gas and a liquid; they show similarities, and we are going to summarize some of them below.

Rotation of the flow (14) is described by its zeroth mode

$v_{0}=\frac{A k \pi \omega}{s\left(k^{2} \pi^{2}+s^{2}+\sigma\right)} J_{1}(r) e^{\sigma \tau} \cos k \pi z$,

$u_{0}=-\frac{A k \pi}{s} J_{1}(r) e^{\sigma \tau} \cos k \pi z$

$w_{0}=A J_{0}(r) e^{\sigma \tau} \sin k \pi z, k= \pm 1, \pm 2, \ldots r=\frac{r^{\prime} s}{h}$.

The stream lines projections of the zeroth mode on the plane $z=$ const are spirals:

$r=C \exp \left(-\left(k^{2} \pi^{2}+s^{2}\right) \frac{\varphi}{\omega}\right)$

Such spirals are very typical for satellite photos of hurricanes, and were also reported by Nalivkin (1986), taking place in the internal tornado cavity (see Fig. 1). Clearly, for the fluid motion without Coriolis force $(\omega=0)$ the solution (15) is not twisted.

The main parameter for convection description is Relay's number. From formulas (13) the threshold value of Relay's number $\vartheta_{1}$ corresponding to beginning of convective motion equals to the minimum of the following expression as a function of parameter $s: \vartheta_{1}(s, \omega)=\left[\left(k^{2} \pi^{2}+s^{2}\right)^{3}+\omega^{2} k^{2} \pi^{2}\right] / s^{2}$. For $\omega=0$ this minimum of the function $\vartheta_{1}(s, \omega)$ is known from the Benard effect theory: $\min \vartheta_{1}(s, 0)=\vartheta *=\frac{27 \pi^{4}}{4} \approx 657.5$. The minimum is achieved at $s^{*}(0)=\frac{\pi}{\sqrt{2}} \approx 2.2$.

Rotation provides stabilizing effect for the convective fluid flow due to $\omega^{2}$ term in $\vartheta_{1}(s, \omega)$, therfore in the rotating fluid layer locally twisted flow (14) starts at larger Relay numbers. This fact is a generalization of the known fact, that spatiallyperiodic perturbations of type $\left\{v_{x}, v_{y}, v_{z}\right\} \sim \exp (\operatorname{im} x+i n y+t)$ can be stabilized in presence of rotation.

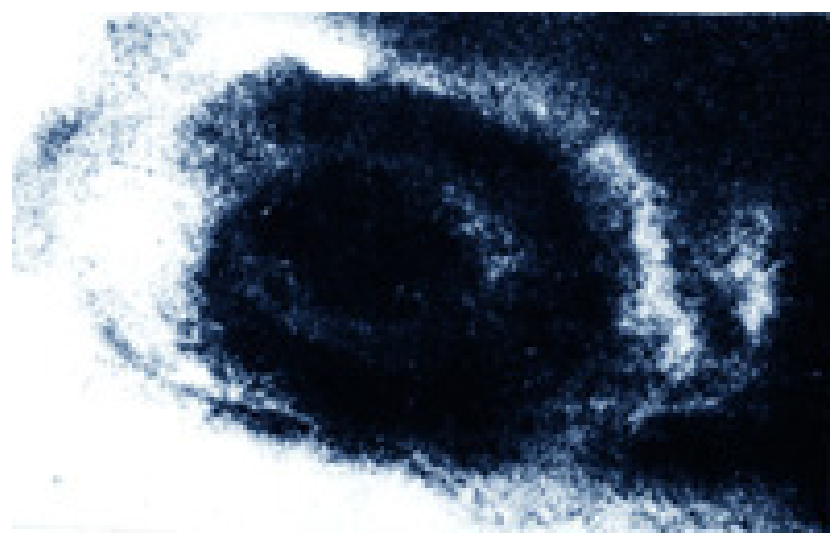

Figure 1. A tornado funnel cavity, view from beneath. The vertical spiral of screw lines in the axis jet can be observed.

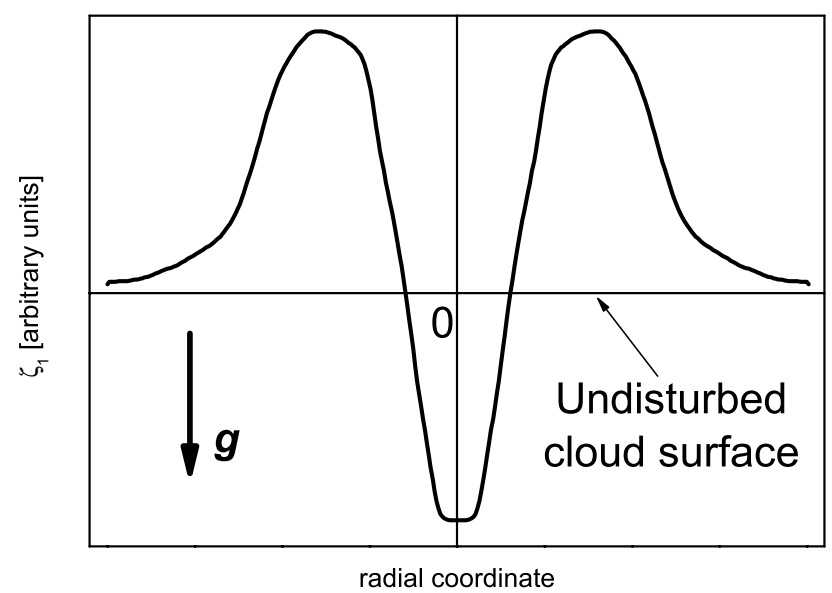

Figure 2. The zeroth mode of the function $\zeta_{1}(r)$ deforming the lower layer boundary.

Hydrodynamic characteristics of the flow (14), (15) disappear with increase of distance from the axis $(r \rightarrow \infty)$.

At $s \rightarrow \infty$ the fluid motion (14) (where the mode number $m$ does not grow) and formulas (15) represent a vortex localized in the vicinity of its axis.

For even values of $k$ the zeroth mode $(m=0)$ of the function $\zeta_{2}$ in the formulas (14) deforms the upper layer boundary in the form of a conic hollow in the flow. Such deformations stimulate funnels of convective type on the water surface.

The zeroth mode of the lower boundary function $\zeta_{1}(r)$ at $A k<0$ deforms the lower boundary layer (Fig. 2). The structure consists of a narrow column along the axis, corresponding to the maximum value of the zeroth Bessel function $J_{0}(0)$, surrounded by a wide cone of the opposite sign. Such structure is typical for tornadoes. 
From Eq. (13) one can obtain the Relay's number for the oscillatory instability of the lower mode $k=1$ :

$\vartheta_{2}=\frac{2(1+\gamma)}{s^{2}}\left[\left(\pi^{2}+s^{2}\right)^{3}+\frac{\pi^{2} \gamma^{2}}{(1+\gamma)^{2}} \omega^{2}\right]$.

The corresponding frequency of the neutral oscillations depend on the Prandtl number $\gamma$ :

$\Omega^{2}=\frac{\pi^{2}(1-\gamma)}{\left(\pi^{2}+s^{2}\right)(1+\gamma)}\left(\omega^{2}-\omega_{*}^{2}\right)$

where $\omega_{*}^{2}=\frac{\left(\pi^{2}+s^{2}\right)^{2}}{\pi^{2}} \frac{1+\gamma}{1-\gamma}$.

According to formula (19), oscillatory instability is impossible for the Prandtl numbers $\gamma>1$.

Similarity of the dispersion relations (13) in the case of vortical and spatially periodic perturbations means that the perturbation type is defined by the wave numbers $m, n$ and $s$, rather than by the temperature regime in the layer or other non-physical properties of the fluid.

If the parameter of voritical perturbations $s$ coincides with the absolute value of wave vector $\boldsymbol{a}$ of the spatially-periodic perturbations, then both scenarios are equiprobable. Otherwise, the flow with higher value of growth rate will dominate. The growth rate itself depends on the positions of the parameters $s$ and $a$ on the wave-numbers axis.

For simplicity, let us restrict ourselves to the case of the non-rotating layer $(\omega=0)$. The growth rate of the most dangerous mode $k=1$ of the vortical perturbations (13) is

$\sigma=\left[\sqrt{\frac{(\gamma-1)^{2}}{4}+\frac{\vartheta s^{2} \gamma}{\left(\pi^{2}+s^{2}\right)^{3}}}-\frac{\gamma+1}{2}\right] \frac{\left(\pi^{2}+s^{2}\right)}{\gamma}$.

The growth rate of the spatially-periodic motion can be obtained from (20) by substituting $s^{2}=a^{2}=m^{2}+n^{2}$. In the limit of large Rayleigh numbers $\vartheta \gg 1$ in (20), the growth rate $\sigma(s)$ reaches zero at

$s=s_{1}=\frac{\pi^{3}}{\sqrt{\vartheta}}+O\left(\frac{1}{\vartheta}\right), \quad s=s_{2}=\sqrt[4]{\vartheta}+O\left(\frac{1}{\sqrt[4]{\vartheta}}\right)$,

and the growth rate $\sigma(s)$ has maximum value at

$s=s^{*}=\sqrt[8]{\frac{\pi^{4} \gamma \vartheta}{(\gamma+1)^{2}}}+O\left(\frac{1}{\sqrt[8]{\vartheta}}\right)$.

In the interval $s_{1}<s<s *$, the growth rate of the vortical perturbations monotonously increases with $s$. If $s$ also exceeds the absolute value of the wave number of spatially-periodic perturbations $(s>a)$, then vortical perturbations prevail over the spatially-periodic ones. For interval $s *<s<s_{2}$ the growth rate (20) decreases monotonously with respect to $s$. In this case, local vortical perturbations will appear if the parameter $s$ of the vortical perturbations is smaller than the spatiallyperiodic wave number: $(s<a)$.
The fact that the vortical perturbations of tornado type are observed in laboratory experiments less frequent than the roll or hexagonal stability losses, is because the layer is more sensitive to the spatially-periodic perturbations at the beginning of the convention flow at $s_{1}<s<s *$.

With increase of $s$ (at $s^{*}<s<s_{2}$ ) the layer becomes more sensitive to vortical perturbations. The main feature of the tornado-type vortices is absence of spatial (radial) periodicity. And the initial perturbations in atmosphere (initial rotation of the cloud, shear air flows, Coriolis effect, instability of the vertical non-rotating flow, etc.) are the perturbations without periodicity in space. Under these conditions, despite of the similarity of the dispersion relations, the vortical structure of instability is more preferable than the periodic in space one, because either spatially-periodical flow is absent, or the layer is more sensitive to the vortical disturbances. This corresponds to the case discussed above $\left(s<a, s^{*}<s<s_{2}\right)$, where the growth rate monotonously decreases with $s$, so that increasing the parameter $s<a$ decreases the growth rate and the perturbation magnitude.

\section{Conclusions}

In this paper we tried to develop a convection model in the cloudy layer and to find out whether the convection in this conditions can be a localized phenomenon. It was found that if a layer has movable boundaries, convection can take place at some point and disappear at long distances. The region where the convection takes place is associated with the larger instability gradients. This region once formed develops and can reach a stationary state. Existence of such a region is associated with the cloud rotation, which should be sufficiently strong. The supercell rotation in the tornados observed in the USA is often associated with the atmosphere jet stream.

Edited by: F. Stel and D. B. Giaiotti

Reviewed by: two anonymous referees

\section{References}

Bergeron, T.: The atmosphere and the sea in motion, edited by: Bolin, B., The Rockefeller Institute Press, 440-474, 1959.

Browning, K. A. and Ludlam, F. H.: Q. J. Roy. Meteor. Soc., 88, 117-135, 1962.

Browning, K. A.: Airflow and Precipitation Trajectories Within Severe Local Storms Which Travel to the Right of the Winds, J. Atmos. Sci., 21, 634-639, 1964.

Chandrasekhar, S.: Hydrodynamic and hydromagnetic stability, Oxford, Clarendon Press, 1961.

Dowell, D. C., Alexander, C. R., Wurman, J. M., and Wicker, L. J.: Centrifuging of Hydrometeors and Debris in Tornadoes: Radar-Reflectivity Patterns and Wind-Measurement Errors, Mon. Weather Rev., 133, 1501-1524, 2005.

Emanuel, K. A. and Bister, M.: Moist Convective Velocity and Buoyancy Scales, J. Atmos. Sci, 53, 3276-3285, 1996. 
Kessler, E.: Tornadoes: state of knowladge, J. Struct. Div. Proc. Amer. Soc. Civ. Eng., 104, 2, 352-357, 1978.

Landau, L. D. and Lifshitz, E. M.: Fluid Mechanics, 2nd Ed., Pergamon Press, 1987.

Nalivkin, D. V.: Hurricanes, Storms and Tornadoes, Balkema, Rotterdam, The Netherlands, 1986.

Renno, N. O. and Ingersoll, A. P.: J. Atmos. Sci., 53 572-583, 1996.

Renno, N. O., Burkett, M. L., and Larkin, M. P.: A Simple Thermodynamical Theory for Dust Devils, J. Atmos. Sci., 55, 32443252, 1998.
Rutkevich, P. B., Tur, A. V., and Yanovsky, V. V.: Internal wavesurface wave interaction in an arbitrary stratified ocean, Izvestija, Atmos. Ocean. Phys., 25, 794-798, 1989.

Souza, E. P., Renno, N. O., and Dias, M. A. F. S.: Convective Circulations Induced by Surface Heterogeneities, J. Atmos. Sci., 57, 2915-2922, 2000.

Wicker, L. J. and Wilhelmson, R. B.: Simulation and Analysis of Tornado Development and Decay within a Three-Dimensional Supercell Thunderstorm, J. Atmos. Sci., 52, 2675-2703, 1995. 